\title{
Interleukin-6 is a Strong Predictor of the Frequency of COPD Exacerbation Within I Year
}

\author{
Hui Huang ${ }^{1-3}$ \\ Xiaodong Huang ${ }^{3}$ \\ Kaojuan Zeng ${ }^{3}$ \\ Fan Deng ${ }^{3}$ \\ Changqing Lin $^{3}$ \\ Wenjie Huang ${ }^{1,2}$
}

'The First School of Clinical Medicine, Southern Medical University, Guangzhou, 5105I5, People's Republic of China;

${ }^{2}$ Department of Respiratory and Critical Care Medicine, General Hospital of Southern Theatre Command, Guangzhou, 510010, People's Republic of China; ${ }^{3}$ Department of Respiratory and Critical Care Medicine, Huizhou Municipal Central Hospital, Huizhou, 5I600I, People's Republic of China
Correspondence: Wenjie Huang

The First School of Clinical Medicine, Southern Medical University, No. 1023 1063, Shatai South Road, Guangzhou, 510010 , People's Republic of China

Tel +86 I3600466358

Fax +8620-88686500

Email huangyelu1029@vip.I63.com
Purpose: Persistent chronic inflammation of chronic obstructive pulmonary disease (COPD) is associated with poor outcomes and frequently results in acute exacerbation. Predicting the number of exacerbations is important. Because interleukin 6 (IL-6) plays an important role in inducing and maintaining chronic inflammation, we sought to observe whether IL-6 measurement can predict the frequency of acute exacerbation of COPD.

Methods: We reviewed serum IL-6 concentrations of stable COPD patients from January 2016 to December 2017 and statistically analyzed them to determine the optimal threshold value to predict the frequency of COPD acute exacerbations. Outpatients with stable COPD were then recruited between January 2018 and December 2019 and grouped into a low IL-6 group and a high IL-6 group according to this threshold value. We then compared the number of exacerbations of COPD in 1 year between the two groups.

Results: We reviewed data from 95 COPD patients, who had a median of 1.00 exacerbations in preceding year; 35 of these patients had no fewer than two. The median IL-6 concentration was $8.80 \mathrm{pg} / \mathrm{mL}$. IL-6 and hs-CRP were positively correlated with frequency of acute exacerbation in the preceding year, COPD assessment test (CAT) score and British medical research council (mMRC) score, and negatively correlated with forced expiratory volume in one second as percentage of predicted value ( $\mathrm{FEV}_{1} \%$ pred) and $\mathrm{FEV}_{1} / \mathrm{FVC} \%$ (forced vital capacity). IL-6 was the risk factor of COPD patients with two or more exacerbations in 1 year. Finally, we enrolled 65 COPD patients and divided into low IL- 6 group and high IL-6 group; the high IL-6 group experienced more frequent exacerbations than did the low IL- 6 group.

Conclusion: An IL-6 measurement of $14.030 \mathrm{pg} / \mathrm{mL}$ or more is a risk factor for $\geq 2$ acute exacerbations of COPD in the following year.

Keywords: hs-CRP, IL-6, risk factor, prediction, stable COPD, biomarkers

\section{Introduction}

Chronic obstructive pulmonary disease (COPD) is a common, preventable, and treatable chronic airway inflammatory disease. COPD was the fifth leading cause of death in China and the fourth leading cause of death globally; it ranked eighth in causes of disease burden as measured by disability-adjusted life years in 2015. ${ }^{1,2}$

COPD was previously considered to primarily affect the lungs, but now it is increasingly accepted that COPD is a systemic inflammation. ${ }^{3}$ Persistent systemic inflammation is associated with poor clinical outcomes in COPD and results in frequent acute exacerbation. ${ }^{4}$ This reduces quality of life, speeds disease progression, and increases the risk of death. Therefore, reducing the number of exacerbations is a primary goal of treatment; ${ }^{5}$ predicting the number of acute exacerbations in the year following diagnosis is of particular importance. 
Interleukin 6 (IL-6) is a soluble inflammatory factor that synthesized in a location in the early stages of inflammation, and then moves to the liver, followed by rapid induction of an extensive range of acute phase proteins such as C-reactive protein (CRP), fibrinogen, and serum amyloid A (SAA). ${ }^{6} \mathrm{IL}-$ 6 plays an important role in inducing and maintaining chronic inflammation. It has been shown that IL-6 induces the differentiation of Th17 combined with transforming growth factor (TGF)- $\beta$, while inhibits differentiation of regulatory $\mathrm{T}$ cells (Treg) induced by TGF- $\beta .^{7}$ And up-regulation of the Th17/ Treg balance is considered to involve in the development of chronic inflammatory. ${ }^{6}$ Overproduction of IL- 6 has been found in many autoimmune and chronic inflammatory disease including rheumatoid arthritis, systemic lupus erythematosus, Crohn's disease, asthma, and other inflammatory pulmonary diseases. ${ }^{7,8}$ IL-6 has also been linked to chronic inflammation-related cancers, such as lung cancer, colorectal cancer, and so on. ${ }^{7}$ Increasing evidence shows that IL-6 levels are associated with COPD: IL-6 levels are increased in induced sputum of COPD patients, and are inversely correlated with lung function measures such as forced expiratory volume in one second as percentage of predicted value $\left(\mathrm{FEV}_{1}\right.$ \%pred). ${ }^{9,10}$ Two 3-year follow-up studies have shown that elevated IL-6 levels in serum were associated with increased mortality in COPD. ${ }^{11,12}$ Increased serum IL-6 levels have also been associated with worse six-minute walking distance (6MWD) performance and poor clinical outcomes in COPD patients. ${ }^{4,12}$ Some studies have suggested that the $-572 \mathrm{C}$ allele in IL-6 reduced the risk of developing COPD, while the IL-6 -745G/C single nucleotide polymorphism (SNP) increased the risk. ${ }^{13}$

In summary, IL-6 in COPD plays an important role in maintaining chronic inflammation, leading to poor outcomes and increasing the risk of death. However, it is not enough evidence to support IL-6 is an excellent predictor in AECOPD, it has not been widely used in clinical. Thus, this study aimed to observe whether IL-6 can predict the frequency of acute exacerbation of COPD through 1-year follow-up of COPD patients, and provide more evidence to support IL-6 is a strong predictor.

\section{Materials and Methods}

\section{Study Design and Population}

First, serum IL-6 concentration levels of stable COPD patients from January 2016 to December 2017 were reviewed and statistically analyzed to obtain the optimal threshold value to predict the frequency of COPD acute exacerbations. Outpatients with stable COPD were then recruited between January 2018 and December 2019 and were grouped into either the low IL-6 group or the high IL-6 group according to this identified optimal threshold value. All recruited patients were followed for 1 year. The study scheme is described in Figure 1.

\section{Participants and Data Collection Procedures in Retrospective Analysis}

All studied subjects were recruited from the Department of Respiratory and Critical Care Medicine at Huizhou Municipal Central Hospital. All patients with COPD met the inclusion criteria: (1) the diagnosis of COPD was based on the Global initiative for Chronic Obstructive Lung Disease (GOLD) guidelines, (2) aged $\geq 40$ years, (3) used inhaled corticosteroids (ICS)/bronchodilators regularly over 1 month, (4) no acute exacerbation required antibiotics and systemic hormones in the preceding 1 month, (5) no other lung disease or uncontrolled comorbidities, and (6) able to sign informed consent and complete the questionnaire.

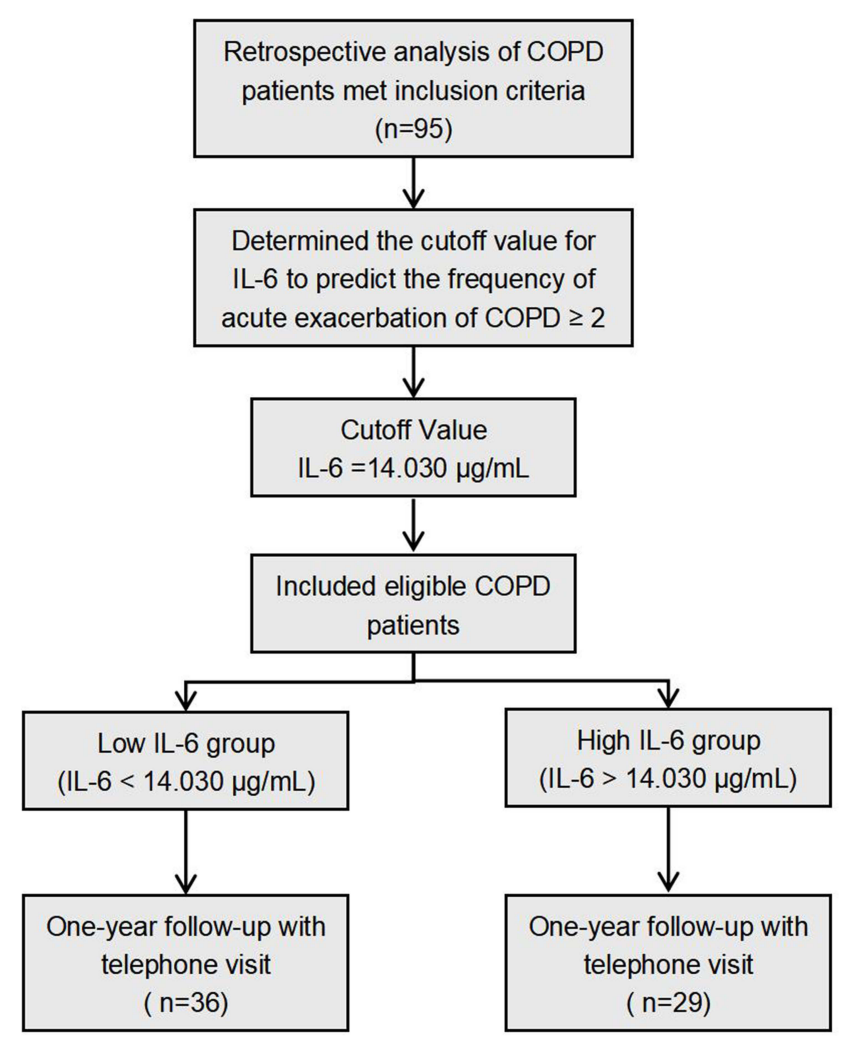

Figure I Study scheme.

Abbreviations: COPD, chronic obstructive pulmonary disease; IL-6, interleukin 6 . 
Data on demographics, smoking status, diagnosis, the frequency of acute exacerbation of COPD in 1 year, lung function, COPD assessment test (CAT), modified British medical research council (mMRC) dyspnea scale, and hematological indicators and treatment were collected from hospital electronic medical record system. Serum IL-6 of enrolled subjects would analyse to obtain the optimal threshold value to predict the frequency of COPD acute exacerbations, and then the value would use in the follow up study.

\section{Participants and Data Collection \\ Procedures in One-Year Follow-Up Study}

The inclusion criteria of studied subjects were similarly with the case review. Lung function, CAT, mMRC and hematological indicators were measured in the first visit. Enrolled patients need to perform outpatient follow-up visit every 3 months to evaluate their condition. When their COPD symptoms exacerbated and need additional medical attention, they should contact the study doctor at any time and visit our hospital in time. Patients in the stable period who did not wish to perform outpatient follow-up visit were conducted telephone follow-up visit every 6 months to inquire about the frequency of acute exacerbation of COPD. In one-year follow-up study period, the frequency of AECOPD was assessed and confirmed according to the medical record and the treatment history.

\section{Outcomes Measurement}

CAT and mMRC were measured when subjects enrolled. Lung function was detected by lung function department in our hospital, and according to the lung function detection guideline. The results of lung function were admitted within 6 months. Hematological indicators comprising complete blood cell count, serum biochemical indexes and serum IL- 6 were measured by the clinical laboratory in our hospital. Serum IL-6 concentration was determined by ELISA kit (PI330) which purchased from Beyotime Biotechnology.

\section{Ethics}

Our study complied with the Declaration of Helsinki Ethical Principles for Medical Research Involving Human Subjects. The study was also approved by the Ethics Committee of Huizhou Central People's Hospital, and informed consent was obtained from all patients before enrollment in the study. The privacy of all patients was protected, and the data was anonymized and maintained with confidentiality.

\section{Statistical Methods}

The statistical analysis was performed using SPSS statistical package (SPSS version 22.0, IBM, Armonk, NY, USA). The normality of data was examined using the Shapiro-Wilk test. Continuous variables with normal distribution are presented as means \pm standard deviation (SD), while continuous variables with non-normal distribution are presented as medians and interquartile range (IQR). Correlations among variables were analyzed using Spearman correlation test. Binary regression analyses were performed to evaluate the potential independent association between IL- 6 and the frequency of acute exacerbation of COPD. The accuracy of IL-6 was assessed with receiver operating curve (ROC) analysis. The chi-squared test was used to compare categorical variables between the low IL6 group and the high IL-6 group. The Mann-Whitney $U$-test was used to compare the non-normal distribution data between different groups. $P<0.05$ was considered statistically significant.

\section{Results}

\section{Patient Characteristics}

We reviewed data from 95 stable COPD patients who presented to our hospital between January 2016 and December 2017. The demographic, clinical, lung function and hematological indicators of patients are summarized in Table 1. The median number of acute exacerbations in the past year in the included COPD patients was 1.00; 35 of these patients had two or more. The overall median serum IL-6 concentration was $8.80 \mathrm{pg} / \mathrm{mL}$.

\section{Correlation Between hs-CRP, IL-6 and Clinical Data}

We analyzed the correlation between hs-CRP, IL-6 and clinical data related to COPD prognosis. The results showed that IL-6 was positively correlated with frequency of acute exacerbation in the previous year, CAT score and mMRC score $(r=0.852,0.850$, and 0.684 , respectively; all $P<0.001$ ), but negatively correlated with $\mathrm{FEV}_{1} \%$ pred and $\mathrm{FEV}_{1} / \mathrm{FVC}_{0}(r=-0.948,-0.522$; both $P<0.001)$. Correlation analysis of hs-CRP also showed similar results, but the degree of correlation was lower than that of IL-6 (Table 2). 
Table I Characteristics of COPD Patients $(n=95)$

\begin{tabular}{|c|c|c|}
\hline Variable & $\begin{array}{l}\text { Mean/N/ } \\
\text { Median }\end{array}$ & $\begin{array}{c}\text { SDI } \\
\% / I Q R\end{array}$ \\
\hline \multicolumn{3}{|l|}{ Demographic } \\
\hline Age (years-old; median, IQR) & 72.00 & 15.00 \\
\hline Male $(\mathrm{N}, \%)$ & 63 & 66.32 \\
\hline BMI $\left(\mathrm{kg} / \mathrm{m}^{2} ;\right.$ median, IQR) & 19.60 & 2.37 \\
\hline Smoking status (N, \%) & & \\
\hline Smoker & 69 & 72.63 \\
\hline Ex-smoker & 12 & 12.63 \\
\hline \multicolumn{3}{|l|}{ Clinical } \\
\hline Course of disease (years; median, IQR) & 10.00 & 5.00 \\
\hline $\begin{array}{l}\text { Frequency of acute exacerbation in the } \\
\text { past I year (median, IQR) }\end{array}$ & 1.00 & 1.00 \\
\hline Exacerbation frequency $\geq 2$ & 35 & 36.84 \\
\hline CAT score (median, IQR) & 18.00 & 7.00 \\
\hline mMRC score (median, IQR) & 2.00 & 1.00 \\
\hline \multicolumn{3}{|l|}{ Lung Function } \\
\hline FEV I (L; median, IQR) & 1.20 & 0.60 \\
\hline FEV $1 \%$ pred (median, IQR) & 57.21 & 16.28 \\
\hline $\mathrm{FEV}_{\mathrm{I}} / \mathrm{FVC} \%$ (mean, SD) & 53.94 & 7.85 \\
\hline \multicolumn{3}{|l|}{ Hematological Indicators } \\
\hline WBC $\left(\times 10^{9} ;\right.$ mean, SD $)$ & 8.26 & 1.97 \\
\hline NE $\left(\times 10^{9} ;\right.$ median, IQR $)$ & 5.63 & 2.28 \\
\hline EO $\left(\times 10^{9} ;\right.$ median, IQR $)$ & 0.10 & 0.41 \\
\hline hs-CRP (mg/L; median, IQR) & 5.50 & 6.64 \\
\hline IL-6 (pg/mL; median, IQR) & 8.80 & 26.07 \\
\hline
\end{tabular}

Notes: Normal range of WBC, NE, EO, hs-CRP and IL-6 $\left(3.5 \sim 9.5 \times 10^{9}\right.$, I.8 6.3 $\left.\times 10^{9}, 0.02 \sim 0.52 \times 10^{9}, 0 \sim 5 \mathrm{mg} / \mathrm{L}, 0 \sim 7 \mathrm{pg} / \mathrm{mL}\right)$.

Abbreviations: IQR, interquartile range (25th-75th percentile); $\mathrm{N}$, number; SD, standard deviation; BMI, body mass index; CAT, chronic obstructive pulmonary disease assessment test; mMRC, modified British medical research council; $\mathrm{FEV}_{1}$, forced expiratory volume in I second; $F E V_{1} \% p r e d, F E V_{1}$ as a percentage of the predicted value; FVC, forced vital capacity; WBC, white blood cell count; NE, absolute neutrophil count; EO, absolute eosinophil count; hs-CRP, hypersensitive C reactive protein; IL-6, interleukin-6.

Table 2 Correlation Between hs-CRP, IL-6 and Clinical Data

\begin{tabular}{|l|c|c|c|c|}
\hline \multirow{2}{*}{ Variables } & \multicolumn{2}{|c|}{ hs-CRP } & \multicolumn{2}{c|}{ IL-6 } \\
\cline { 2 - 5 } & $\boldsymbol{r}$ & $P$ & $\boldsymbol{r}$ & $\boldsymbol{P}$ \\
\hline Frequency of acute & 0.572 & 0.000 & 0.852 & 0.000 \\
exacerbation in the past I year & & & & \\
CAT score & 0.559 & 0.000 & 0.850 & 0.000 \\
mMRC score & 0.363 & 0.000 & 0.684 & 0.000 \\
FEVI\%pred & -0.556 & 0.000 & -0.948 & 0.000 \\
FEVI/FVC\% & -0.388 & 0.000 & -0.522 & 0.000 \\
\hline
\end{tabular}

Note: $P<0.05$ considered significant.

Abbreviations: CAT, chronic obstructive pulmonary disease assessment test; mMRC, modified British medical research council; $\mathrm{FEV}_{1}$, forced expiratory volume in I second; FEV $\%$ pred, FEV as a percentage of the predicted value; FVC, forced vital capacity; hs-CRP, hypersensitive C reactive protein; IL-6, interleukin-6.

\section{Risk Factors for $\geq 2$ Acute Exacerbations of COPD}

The frequency of acute exacerbation of COPD is associated with patients' prognosis. Two or more exacerbations per year is one of the criteria for the comprehensive assessment of COPD in groups $\mathrm{C}$ and $\mathrm{D}$ of the GOLD classification, which often indicates more severe disease status. Because our results showed that IL- 6 and hs-CRP correlated with frequency of acute exacerbation of COPD (Table 2), we analyzed these data using binary regression analysis to identify the risk factors for two or more acute exacerbations of COPD. The results suggested that higher IL-6 measurements were significantly associated with a higher risk of having two or more acute exacerbation within 1 year (OR $0.260,95 \%$ CI $1.140-1.475$ ), indicating that IL-6 measurements were capable of predicting the frequency of exacerbation (Table 3).

\section{ROC Curve Analysis of IL-6 in Predicting the Frequency of Acute Exacerbation}

The validity of IL-6 in predicting two or more acute exacerbations of COPD was excellent and significant; the area under the curve (AUC) was $0.970 \quad(P<0.001)$ (Figure 2). A cut-off value of IL-6 $>14.030 \mathrm{pg} / \mathrm{mL}$ had $88.6 \%$ sensitivity and $95 \%$ specificity.

\section{Frequency of Acute Exacerbation by Group After I-Year Follow-Up}

Using this cut-off value, eligible COPD patients were divided into a low IL-6 group (IL-6 $<14.030 \mathrm{pg} / \mathrm{mL}$ ) and a high IL-6 group (IL-6 $>14.030 \mathrm{pg} / \mathrm{mL}$ ). There were no differences between the two groups in terms of demographic and lung function data, CAT score, or mMRC score (Table 4). The frequency of acute exacerbation events in 1 year was higher in the high IL-6 group than in the low IL-6 group ( 2.00 vs $1.00, P<0.001)$, and those patients who had $\geq 2$ exacerbation events were more likely to be in the high IL-6 group $(79.31 \%$ vs $38.89 \%$, $P=0.001)$.

Table 3 Risk Factors of the Frequency of Acute Exacerbation of COPD $\geq 2$ Using Binary Regression Analysis

\begin{tabular}{|l|l|l|l|}
\hline Variables & OR & $\mathbf{9 5 \%} \mathbf{~ C l}$ & $\boldsymbol{P}$ \\
\hline hs-CRP & 0.110 & $0.901-1.382$ & 0.313 \\
IL-6 & 0.260 & $1.140-1.475$ & 0.000 \\
\hline
\end{tabular}

Abbreviations: hs-CRP, hypersensitive C reactive protein; IL-6, interleukin-6; OR, odds ratio; $\mathrm{Cl}$, confidence interval. 


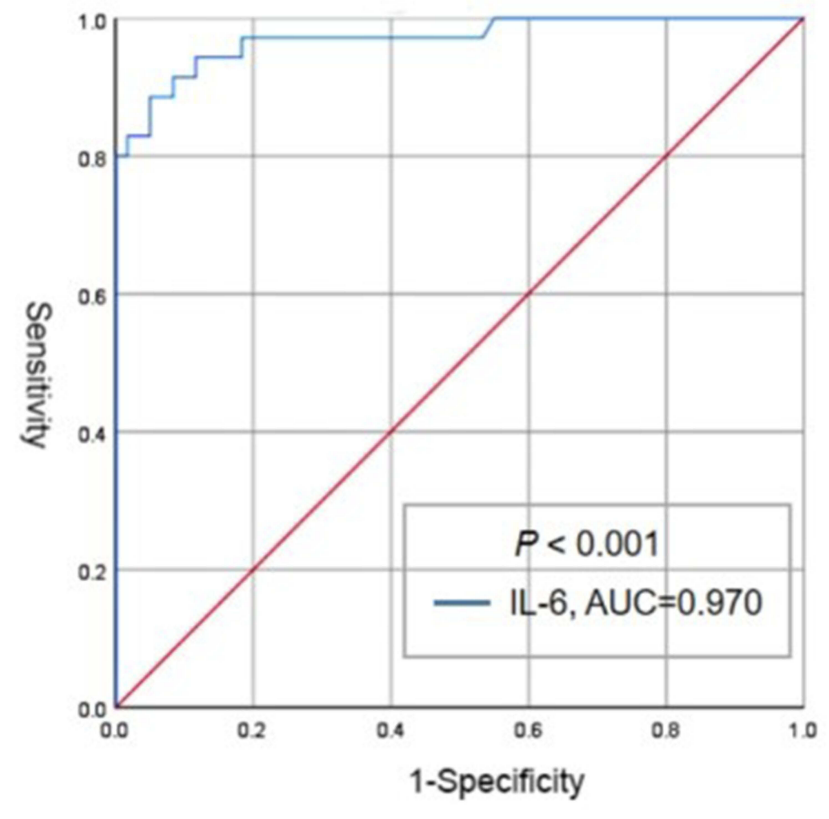

Figure 2 ROC analysis of IL- 6 in predicting the frequency of acute exacerbation of COPD $\geq 2$.

Abbreviations: IL-6, interleukin 6; AUC, the area under the curve.

\section{Discussion}

COPD is now recognized as a chronic systemic inflammatory disease. Persistent systemic inflammation has been found to be associated with exacerbation frequency in COPD. ${ }^{4}$ Frequent acute exacerbation of COPD not only affects patients' quality of life, but also greatly shortens their life expectancy. ${ }^{14}$ Predicting the number of acute exacerbations in the following year is important because of the impact of these events on the natural history of COPD. ${ }^{5}$ IL-6 represents a keystone cytokine in inflammation which drives progression, and it is now regarded as a prominent target for clinical intervention. ${ }^{15,16}$ Increasing evidence shows that IL-6 is also increased in COPD patients and associated with the progression of COPD. ${ }^{17}$

In the present study, we reviewed the serum IL-6 concentration and clinical data of 95 stable COPD patients at our hospital. The median serum IL-6 concentration in these patients was $8.80 \mathrm{pg} / \mathrm{mL}$, between the results found by Zeng $(30.5 \pm 9.9 \mathrm{pg} / \mathrm{mL})$ and by Gorska $(3.4 \mathrm{pg} / \mathrm{mL}) .{ }^{18,19}$ The difference in results may be due to differences in the severity of COPD patients included, or to differences in suppliers of machines or reagents that measured IL-6 concentration. Most COPD patients included in our study had moderate-to-severe COPD (median $\mathrm{FEV}_{1}$ \%pred was $57.21 \%$ ), but Zeng's patients had more severe COPD (mean $\mathrm{FEV}_{1}$ \%pred was 45.2\%) and Gorska's patients had more mild-to-moderate COPD (median $\mathrm{FEV}_{1} \%$ pred was $71 \%$ ). ${ }^{18,19}$ At present, there are no evidence-based studies with sufficient sample size to confirm serum IL-6 concentration in patients with COPD. Our results exceeded the normal range $(0-7 \mathrm{pg} / \mathrm{mL})$, which may indicate that most COPD patients had chronic systemic inflammation. Because one major effect of IL-6

Table 4 Compared the Frequency of Acute Exacerbation Between Different Groups After One-Year Follow-Up

\begin{tabular}{|c|c|c|c|}
\hline Variables & Low IL-6 Group $(n=36)$ & High IL-6 Group $(n=29)$ & $P$ value \\
\hline \multicolumn{4}{|l|}{ Demographic } \\
\hline Age (years-old; mean, SD) & $67.97 \pm 1.47$ & $68.27 \pm 1.58$ & 0.864 \\
\hline Male $(\mathrm{N}, \%)$ & $25,69.44$ & $19,65.52$ & 0.736 \\
\hline BMI $\left(\mathrm{kg} / \mathrm{m}^{2} ;\right.$ mean, SD or median, IQR $)$ & $20.40 \pm 0.33$ & $21.71,5.32$ & 0.078 \\
\hline Smoker (N, \%) & 3I, 86.II & $22,75.86$ & 0.290 \\
\hline \multicolumn{4}{|l|}{ Clinical } \\
\hline Frequency of acute exacerbation in I year (median, IQR) & $1.00,1.00$ & $2.00,1.00$ & 0.000 \\
\hline Exacerbation frequency $\geq 2(\mathrm{~N}, \%)$ & 14, 38.89 & $23,79.31$ & 0.001 \\
\hline CAT Score (mean, SD) & $19.77 \pm 0.54$ & $20.14 \pm 0.66$ & 0.735 \\
\hline mMRC Score (median, IQR) & $2.00,1.00$ & $3.00,1.00$ & 0.268 \\
\hline \multicolumn{4}{|l|}{ Lung Function } \\
\hline FEV,$\% p r e d$ (mean, SD) & $42.46 \pm 1.81$ & $40.04 \pm 1.90$ & 0.391 \\
\hline $\mathrm{FEV}_{\mathrm{I}} / \mathrm{FVC} \%$ (mean, SD) & $49.00 \pm 1.31$ & $48.75 \pm 1.30$ & 0.838 \\
\hline \multicolumn{4}{|l|}{ Hematological Indicators } \\
\hline IL-6 (pg/mL; median, IQR) & $6.73,0.62$ & $32.07,26.67$ & 0.000 \\
\hline
\end{tabular}

Abbreviations: IQR, interquartile range (25th-75th percentile); N, number; SD, standard deviation; BMI, body mass index; CAT, chronic obstructive pulmonary disease assessment test; mMRC, modified British medical research council; $\mathrm{FEV}_{1}$, forced expiratory volume in I second; $\mathrm{FEV}, \%$ pred, $\mathrm{FEV}$, as a percentage of the predicted value; FVC, forced vital capacity; IL-6, interleukin-6. 
is to stimulate the production of CRP, we also evaluated hs-CRP concentration. Hs-CRP is also used as a marker of systemic inflammation in COPD. ${ }^{20}$ In patients with stable stage 2 and stage 3 COPD, hs-CRP concentrations have been reported at median levels of $5.596 \pm 0.7982 \mathrm{mg} /$ $\mathrm{L}$ and $5.093 \pm 0.7912 \mathrm{mg} / \mathrm{L}$, respectively. ${ }^{21}$ Our data showed a similar result; median hs-CRP was $5.50 \mathrm{mg} / \mathrm{L}$, which exceeded the normal range $(0-5 \mathrm{mg} / \mathrm{L})$. Because we included stable COPD patients who regularly used ICS or/and bronchodilators, other hematological indicators such as white blood cells (WBC), neutrophils (NE), and eosinophils (EO) were within normal limits. Some studies have also reported that WBC, NE, and EO were normal in stable COPD patients. ${ }^{22-24}$

Airway remodeling, one of the most prominent causes of airway obstruction in COPD, is often considered the result of chronic inflammation. It has been associated with decreases in lower lung function and frequent exacerbations of COPD. ${ }^{25}$ We analyzed the correlation between hs-CRP, IL-6 and clinical data from COPD patients, and found IL-6 and hs-CRP to be positively correlated with frequency of acute exacerbation in the preceding 1 year, CAT score, and mMRC score, and negatively correlated with FEV1\%pred and $\mathrm{FEV} 1 / \mathrm{FVC} \%$. These findings suggest that elevated levels of IL-6 and hs-CRP are related to poor outcomes from COPD. Binary regression analysis showed that elevated IL-6 (but not hs-CRP) was a risk factor for having two or more exacerbations of COPD within 1 year. CRPgene expression has been shown to be regulated through IL6 independent or dependent pathways, and the secretion of CRP requires induction of IL- $6 .{ }^{26}$ We therefore speculate that IL-6 may better reflect the level of systemic inflammation in COPD patients compared with hs-CRP, potentially explaining why IL-6 was more effective in predicting the occurrence of two or more exacerbation events within 1 year. Based on ROC curve and AUC, the optimal prediction threshold for IL-6 was $14.030 \mathrm{pg} / \mathrm{mL}$.

To further confirm the predictive power of IL- 6 and to reduce the influence of confounding factors, we matched patients for age, sex, smoking status, CAT score, mMRC score and lung function at the time of inclusion. Finally, we enrolled 65 COPD patients and divided into low IL-6 group and high IL-6 group according to the predictive threshold for IL-6. We found more frequent exacerbations and a greater proportion of patients with multiple exacerbations (79.31\% vs $38.89 \%$ ) in the high IL-6 group than in the low IL-6 group.

The findings of the present study not only enhance the previous evidence on the relationship between IL-6 and outcomes of COPD, but also showed IL- 6 to be a predictor of the frequency of acute exacerbation of COPD. Our study also has some limitations. The sample used for the retrospective analysis to obtain the predictive threshold of IL-6 was from a single center, and the sample size was small. Therefore, the predicted value obtained may not be applicable to all COPD patients. Likewise, the sample size used to verify the predictive power of IL-6 was limited. Although this study had some limitations, it also confirms the value of IL-6 in predicting the frequency of acute exacerbation of COPD, which has clinical significance for the prevention or early detection of susceptibility to these exacerbations.

\section{Limitation}

Our findings were based on a single center, the insufficient sample size was a objective fact and limitation of this study, which may affect the stability of the results. And it requires further verification through multi-center cooperation to expand the sample size.

\section{Conclusion}

Frequent exacerbations of COPD that are associated with persistent chronic systemic inflammation result in poor quality of life and shortened life expectancy. Measurement of IL-6 can help to predict the number of potential exacerbation events in the following year and guide patients or doctors in taking timely and appropriate steps to prevent occurrence of these events.

\section{Acknowledgments}

The authors would like to thank the staff of the Department of Respiratory and Critical Care Medicine in Huizhou Municipal Central Hospital for providing and collecting all the clinical data. We also thank John Daniel from Liwen Bianji (Edanz) for editing the English text of a draft of this manuscript.

\section{Disclosure}

The authors declare no conflicts of interest in this study.

\section{References}

1. Lopez-Campos JL, Soler-Cataluna JJ, Miravitlles M. Global strategy for the diagnosis, management, and prevention of chronic obstructive lung disease 2019 report: future challenges. Arch Bronconeumol. 2020;56(2):65-67. doi:10.1016/j.arbres.2019.06.001

2. Collaborators GBDCoD. Global, regional, and national age-sex specific mortality for 264 causes of death, 1980-2016: a systematic analysis for the Global Burden of Disease Study 2016. Lancet. 2017;390 (10100):1151-1210. 
3. Disease GIfCOL. Global strategy for the diagnosis, management, and prevention of chronic obstructive pulmonary disease 2020 report. Available from: https:/goldcopd.org/gold-reports/. Accessed November 9, 2019.

4. Agusti A, Edwards LD, Rennard SI, et al. Persistent systemic inflammation is associated with poor clinical outcomes in COPD: a novel phenotype. PLoS One. 2012;7(5):e37483. doi:10.1371/journal.pone. 0037483

5. Decramer M, Janssens W, Miravitlles M. Chronic obstructive pulmonary disease. Lancet. 2012;379(9823):1341-1351. doi:10.1016/ S0140-6736(11)60968-9

6. Tanaka T, Narazaki M, Kishimoto T. IL-6 in inflammation, immunity, and disease. Cold Spring Harb Perspect Biol. 2014;6(10):a016295. doi:10.1101/cshperspect.a016295

7. Ataie-Kachoie P, Pourgholami MH, Morris DL. Inhibition of the IL-6 signaling pathway: a strategy to combat chronic inflammatory diseases and cancer. Cytokine Growth Factor Rev. 2013;24(2):163-173. doi:10.1016/j.cytogfr.2012.09.001

8. Rincon M, Irvin CG. Role of IL-6 in asthma and other inflammatory pulmonary diseases. Int J Biol Sci. 2012;8(9):1281-1290. doi:10. $7150 /$ ijbs. 4874

9. Donaldson GC, Seemungal TA, Patel IS, et al. Airway and systemic inflammation and decline in lung function in patients with COPD. Chest. 2005;128(4):1995-2004. doi:10.1378/chest.128.4.1995

10. Grubek-Jaworska H, Paplinska M, Hermanowicz-Salamon J, et al. IL-6 and IL-13 in induced sputum of COPD and asthma patients: correlation with respiratory tests. Respiration. 2012;84(2):101-107. doi: $10.1159 / 000334900$

11. Celli BR, Locantore N, Yates J, et al. Inflammatory biomarkers improve clinical prediction of mortality in chronic obstructive pulmonary disease. Am J Respir Crit Care Med. 2012;185 (10):1065-1072. doi:10.1164/rccm.201110-1792OC

12. Ferrari R, Tanni SE, Caram LM, Correa C, Correa CR, Godoy I. Three-year follow-up of interleukin 6 and C-reactive protein in chronic obstructive pulmonary disease. Respir Res. 2013;14:24. doi:10.1186/1465-9921-14-24

13. Wu X, Yuan B, Lopez E, Bai C, Wang X. Gene polymorphisms and chronic obstructive pulmonary disease. J Cell Mol Med. 2014;18 (1):15-26. doi:10.1111/jcmm.12159

14. Pavord ID, Jones PW, Burgel PR, Rabe KF. Exacerbations of COPD. Int J Chron Obstruct Pulmon Dis. 2016;11(Spec Iss):21-30.

15. Hunter CA, Jones SA. IL-6 as a keystone cytokine in health and disease. Nat Immunol. 2015;16(5):448-457. doi:10.1038/ni.3153
16. Unver N, McAllister F. IL-6 family cytokines: key inflammatory mediators as biomarkers and potential therapeutic targets. Cytokine Growth Factor Rev. 2018;41:10-17. doi:10.1016/j.cytogfr.2018. 04.004

17. Su B, Liu T, Fan H, et al. Inflammatory markers and the risk of chronic obstructive pulmonary disease: a systematic review and meta-analysis. PLoS One. 2016;11(4):e0150586. doi:10.1371/journal.pone.0150586

18. Zeng YY, Hu WP, Zuo YH, Wang XR, Zhang J. Altered serum levels of type I collagen turnover indicators accompanied by IL-6 and IL-8 release in stable COPD. Int J Chron Obstruct Pulmon Dis. 2019;14:163-168. doi:10. 2147/COPD.S188139

19. Gorska K, Nejman-Gryz P, Paplinska-Goryca M, Korczynski P, Prochorec-Sobieszek M, Krenke R. Comparative Study of IL-33 and IL-6 levels in different respiratory samples in mild-to-moderate asthma and COPD. COPD. 2018;15(1):36-45. doi:10.1080/15412 555.2017 .1416074

20. Ghobadi H, Fouladi N, Beukaghazadeh K, Ansarin K. Association of high sensitive CRP level and COPD assessment test scores with clinically important predictive outcomes in stable COPD patients. Tanaffos. 2015;14(1):34-41.

21. Nillawar AN, Bardapurkar JS, Bardapurkar SJ. High sensitive C-reactive protein as a systemic inflammatory marker and LDH-3 isoenzyme in chronic obstructive pulmonary disease. Lung India. 2012;29(1):24-29. doi:10.4103/0970-2113.92358

22. Koo HK, Kang HK, Song P, Park HK, Lee SS, Jung H. Systemic white blood cell count as a biomarker associated with severity of chronic obstructive lung disease. Tuberc Respir Dis. 2017;80(3):304-310.

23. Bafadhel M, Pavord ID, Russell REK. Eosinophils in COPD: just another biomarker? Lancet Respir Med. 2017;5(9):747-759. doi:10.1016/S2213-2600(17)30217-5

24. Bilir B, Altıntaş N, Aydın M, Oran M, Özsu S, Tutar Ü. The predictive role of neutrophil to lymphocyte ratio in chronic obstructive pulmonary disease. Eur J Gen Med. 2016;13(2):105-110.

25. Hirota N, Martin JG. Mechanisms of airway remodeling. Chest. 2013;144(3):1026-1032. doi:10.1378/chest.12-3073

26. Luna JM, Moon YP, Liu KM, et al. High-sensitivity C-reactive protein and interleukin-6-dominant inflammation and ischemic stroke risk: the northern Manhattan study. Stroke. 2014;45(4):979-987. doi:10.1161/STROKEAHA.113.002289

\section{Publish your work in this journal}

The International Journal of COPD is an international, peer-reviewed journal of therapeutics and pharmacology focusing on concise rapid reporting of clinical studies and reviews in COPD. Special focus is given to the pathophysiological processes underlying the disease, intervention programs, patient focused education, and self management protocols. This journal is indexed on PubMed Central, MedLine and CAS. The manuscript management system is completely online and includes a very quick and fair peer-review system, which is all easy to use. Visit http://www.dovepress.com/testimonials.php to read real quotes from published authors. 